\title{
Fighting systematics for Charm CPV at Belle II
}

\author{
Youngjoon Kwon*t \\ Department of Physics, Yonsei University \\ E-mail: yjkwon63@yonsei.ac.kr
}

One of the important questions for the Belle II experiment is the CP violation in the charm sector. In the Standard Model, charm CP violation is expected to be very small, $\mathscr{O}\left(10^{-2}-10^{-3}\right)$. Therefore, observation of charm CP violation beyond this range can be a clear signal of new physics. In this talk, we present the efforts by Belle II in the studies of charm CP violation, trying to reduce the systematic uncertainties and to increase an effective sample size. The main features of Belle II along with its initial performances will be discussed. Then studies of time-integrated and time-dependent charm CP violations are discussed with expectations for Belle II luminosity target.

18th International Conference on B-Physics at Frontier Machines - Beauty2019 -

29 September $/ 4$ October, 2019

Ljubljana, Slovenia

\footnotetext{
${ }^{*}$ Speaker.

${ }^{\dagger}$ Representing the Belle II collaboration; supported by NRF of Korea, with Grant No. 2016R1D1A1B01010135.
} 


\section{Introduction}

Belle II is an experiment where $7 \mathrm{GeV} e^{-}$beam collide with $4 \mathrm{GeV} e^{+}$beam, at the $\mathrm{Su}-$ perKEKB collider, in KEK, Japan. As a predecessor of Belle II, the Belle experiment has collected $e^{+} e^{-}$collision data with an integrated luminosity $\mathscr{L}_{\text {int }}=1039 \mathrm{fb}^{-1}$ and contributed significantly to understanding flavor physics in $B$, charm and $\tau$ systems, and especially observing CP violation in $B$ mesons [1].

Even with a great success of Belle (and BaBar, too), however, our understanding of matterantimatter asymmetry in Universe is still far from complete. Moreover, there are several questions remaining unanswered in the flavor sector of particle physics. For instance, recently $B$-factory experiments such as Belle, BaBar, and LHCb have reported several puzzling results including the so-called $R\left(D^{(*)}\right)$ and $R_{K^{(*)}}$ anomalies. In order to tackle these questions, Belle II experiment has been built and started taking collision data since 2018 .

One of the important questions for Belle II is the CP violation in the charm sector. In the Standard Model (SM), charm CP violation is expected to be very small, $\mathscr{O}\left(10^{-2}-10^{-3}\right)$. Therefore, observation of charm $\mathrm{CP}$ violation beyond this range can be a clear signal of new physics (NP) beyond the SM. In this talk, we present the efforts by Belle II in the studies of charm CP violation, trying to reduce the systematic uncertainties and to increase an effective sample size. The main features of Belle II along with its initial performances will be discussed in Section 2. Then in Section 3, studies of time-integrated charm CP violations are discussed, while Section 4 describes the efforts for time-dependent charm CP violation measurements.

\section{Improvements of Belle II over Belle}

\subsection{Detector performance}

The design goal of peak instantaneous luminosity for Belle II is $\mathscr{L}=8 \times 10^{35} \mathrm{~cm}^{-2} \mathrm{~s}^{-1}$, with the target $\mathscr{L}_{\text {int }}=50 \mathrm{ab}^{-1}$, which are approximately 40 and 50 times that of Belle, respectively. To cope with the high luminosity environment and high event rate, many parts of the Belle II detector are upgraded. For example, vertex measurements have been significantly improved with the innermost two-layers of pixel detector surrounded by four layers of silicon strip detectors. Charged hadron identification is also much improved with the newly installed imaging-Time-ofPropagation detector. The improved performance of Belle II has been confirmed with the data from the initial operations (Phase 2 in 2018, and Phase 3 in 2019). Figure 1 shows the measurement of $D^{0}$ lifetime using the $D^{*+} \rightarrow D^{0} \pi^{+}$decays. The measured value $\tau_{D^{0}}=370 \pm 40 \mathrm{fs}$ is consistent with the world-average value, $410 \mathrm{fs}[2]$.

\subsection{Flavor tagging}

In case a $D$ meson decay final state is a CP eigenstate, e.g. $D^{0} \rightarrow K^{+} K^{-}$, it is necessary to tag the flavor of the parent $D$ meson in order to study the CP asymmetry. Moreover, flavor tagging can result in cleaning up the signal $D$ meson candidates, by removing combinatorial background. At Belle II, we adopt two methods for $D^{0}$ meson tagging: (i) conventional $D^{*}$ tagging (the so called "golden method"), and (ii) Rest-of-event (ROE) tagging. 

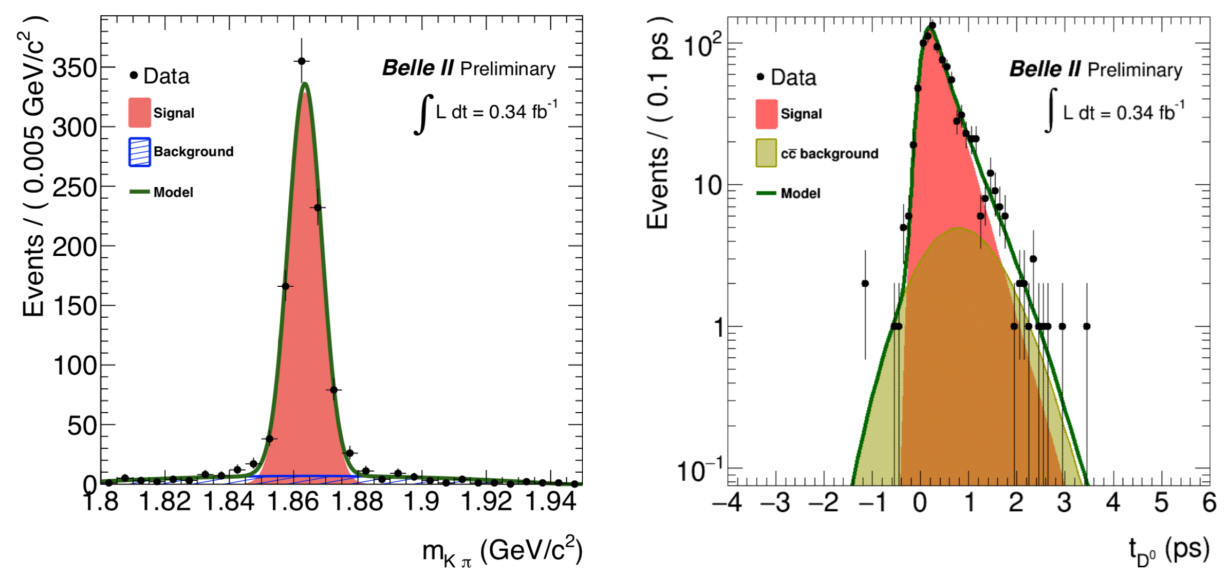

Figure 1: [Left] Invariant mass distribution of the $D^{0}$ candidates tagged by $D^{*+}$ decay; [Right] Decay time distribution of the $D^{0}$ candidates.

The conventional $D^{*}$ tagging uses the decay chain $D^{*+} \rightarrow D^{0} \pi_{\mathrm{s}}^{+}$and we use the variables $M\left(D^{0}\right)$, the invariant mass of the reconstructed $D^{0}$ candidate, and $\Delta M$, the mass difference of the reconstructed $D^{*+}$ and the $D^{0} . \Delta M$ resolution determined from Belle II Monte Carlo (MC) sample is $\sim 180 \mathrm{keV} / c^{2}$ which is better than that of Belle by a factor of two.

Of the $D^{0}$ mesons produced in the process $e^{+} e^{-} \rightarrow c \bar{c}$, only $1 / 4$ arise from $D^{*+}$ decays, while $40 \%$ are produced directly in fragmentation, and the remaining portion is from $D^{* 0}$ decays [3]. In other words, the non- $D^{*+}$ source contributes 3 times more $D^{0}$ mesons than those from $D^{*+}$ decays. The ROE tagging is devised to increase the number of tagged $D^{0}$ candidates for $\mathrm{CP}$ asymmetry study. Figure 2 shows the schematic diagram displayig the principle of the ROE tagging. Once a $D^{0}$ candidate is reconstructed, the rest of the event (hence the name 'ROE') is inspected. Only events with a single $K^{ \pm}$in the ROE are selected. Flavor of the signal $D^{0}$ is determined by the charge of the kaon: a $K^{+}$in the ROE tags the signal side as a $D^{0}$, while a $K^{-}$tags a $\bar{D}^{0}$. With a typical criteria being applied, the ROE tag results in the efficiency $\varepsilon=80 \%$ and the wrong tagging rate $w=13.3 \%$, thus resulting in the effective tagging efficiency $Q_{\mathrm{ROE}}=20.1 \%$ [3]. While this seems low compared to nearly $80 \%$ effective tagging efficiency of the $D^{*+}$ tagging $\left(Q_{D^{*}}\right)$, the lower efficiency is compensated by the higher production rate.

The impact of ROE tagging on the measurement of $\mathrm{CP}$ asymmetry $\left(A_{\mathrm{CP}}\right)$ is estimated by Belle II MC samples. Let $\alpha$ be the ratio of the statistical uncertainty $\sigma_{A_{\mathrm{CP}}}^{\mathrm{ROE}}$ of $A_{\mathrm{CP}}$ with the ROE tag, with respect to that of the $D^{*} \operatorname{tag}, \sigma_{A_{\mathrm{CP}}}^{D^{*}}$ :

$$
\alpha \equiv \frac{\sigma_{A_{\mathrm{CP}}}^{\mathrm{ROE}}}{\sigma_{A_{\mathrm{CP}}}^{D^{*}}}=\sqrt{\frac{1}{3} \frac{Q_{D^{*}}}{Q_{\mathrm{ROE}}} \frac{\rho_{D^{*}}}{\rho_{\mathrm{ROE}}},}
$$

where the factor $1 / 3$ is the production ratio, and $\rho_{D^{*}}$ and $\rho_{\mathrm{ROE}}$ are the purity of the ROE- and $D^{*}$ tagged samples, respectively. We take $\rho_{D^{*}} / \rho_{\mathrm{ROE}} \approx 1.4$, as determined for the BaBar experiment [4]. Given $\alpha$, the significance $\sigma_{A_{\mathrm{CP}}}^{\mathrm{c}}$, for the case when both ROE and $D^{*}$ tagging are combined, can be calculated as $\sigma_{A_{\mathrm{CP}}}^{\mathrm{c}}=\left(\alpha / \sqrt{1+\alpha^{2}}\right) \sigma_{A_{\mathrm{CP}}}^{D^{*}}$. Figure 3 shows the trend of $\sigma_{A_{\mathrm{CP}}}^{\mathrm{c}} / \sigma_{A_{\mathrm{CP}}}^{D^{*}}$ as a function of $\rho_{D^{*}} / \rho_{\mathrm{ROE}}[3]$. We see that roughly a $15 \%$ improvement in the $\sigma_{A_{\mathrm{CP}}}^{\mathrm{c}}$ is expected. 


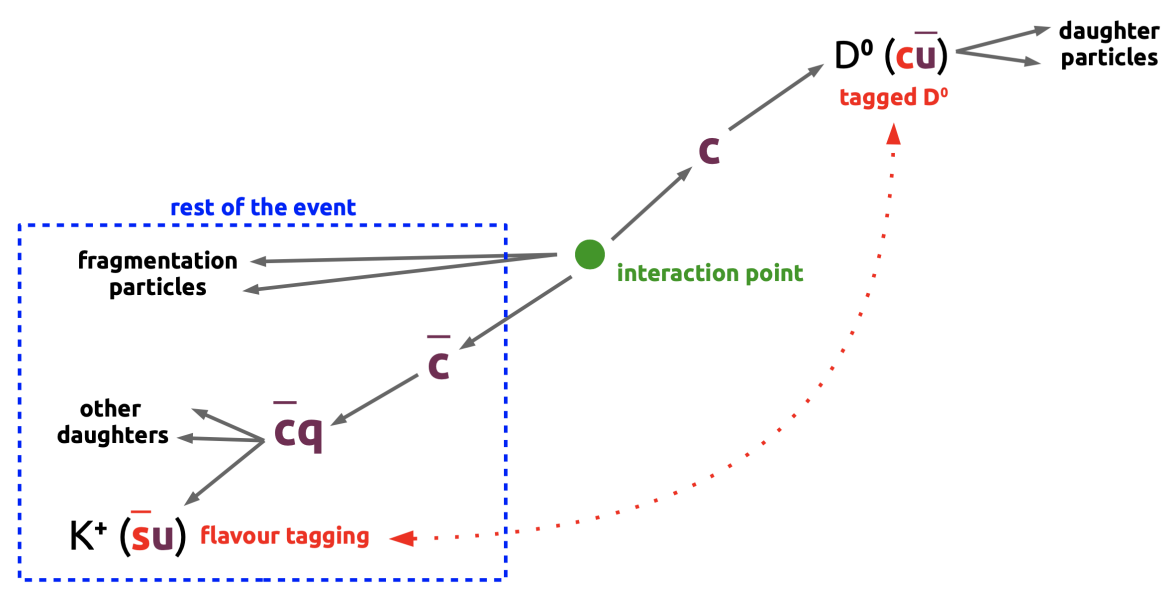

Figure 2: The conceptual diagram of the ROE tagging method. The events with a single $K^{ \pm}$in the ROE are selected, and the flavor of the neutral D meson is determined by the charge of the kaon.

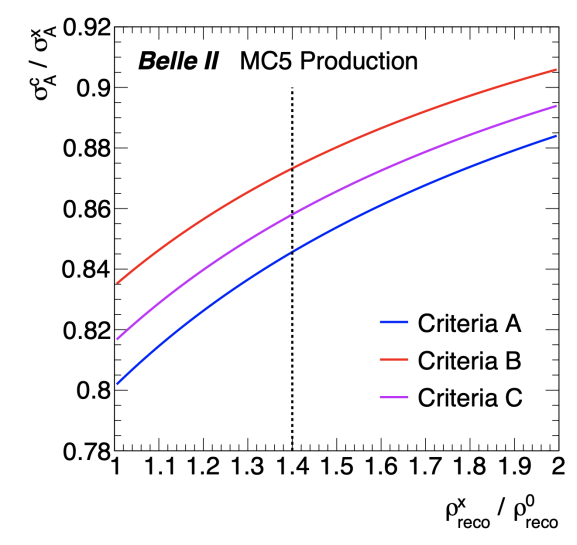

Figure 3: The trend of $\sigma_{A_{\mathrm{CP}}}^{\mathrm{c}} / \sigma_{A_{\mathrm{CP}}}^{D^{*}}$ as a function of $\rho_{D^{*}} / \rho_{\mathrm{ROE}}$. The vertical dotted line corresponds to $\rho_{D^{*}} / \rho_{\mathrm{ROE}}=1.4$ [4]. Criteria A-C are explained in Ref. [3].

\section{Time-integrated charm CP violation}

The time-integrated CP asymmetry $A_{\mathrm{CP}}$ of a charm meson $\left(D^{0}\right.$ or $\left.D^{+}\right)$decaying to a final state $f$ is defined as

$$
A_{\mathrm{CP}} \equiv \frac{\mathscr{B}(D \rightarrow f)-\mathscr{B}(\bar{D} \rightarrow \bar{f})}{\mathscr{B}(D \rightarrow f)+\mathscr{B}(\bar{D} \rightarrow \bar{f})}
$$

If the final state $f$ is flavor-specific, the decay is 'self-tagged', and no tagging is necessary. Otherwise, the aforementioned tagging algorithms (ROE or $D^{*}$ tagging) should be used. To determine $A_{\mathrm{CP}}$, however, one should first measure the raw asymmetry $A_{\text {raw }}$ :

$$
A_{\text {raw }} \equiv \frac{N(D \rightarrow f)-N(\bar{D} \rightarrow \bar{f})}{N(D \rightarrow f)+N(\bar{D} \rightarrow \bar{f})}
$$

The raw asymmetry is then corrected for $D$ production asymmetry $\left(A_{D}\right)$ and the efficiency asymmetry $\left(A_{\varepsilon}\right): A_{\text {raw }}=A_{D}+A_{\varepsilon}+A_{\mathrm{CP}}$. The asymmetry $A_{D}$, arising from the forward-backward asymme- 
try of $D$ production $\left(A_{\mathrm{FB}}^{D}\right)$ can be determined by $A_{\mathrm{FB}}^{D}=\left[A_{\text {raw }}^{\text {corr }}\left(\cos \theta^{*}\right)-A_{\text {raw }}^{\text {corr }}\left(-\cos \theta^{*}\right)\right] / 2$, while $A_{\mathrm{CP}}$ is independent of $\cos \theta^{*}$, hence $A_{\mathrm{CP}}=\left[A_{\text {raw }}^{\text {corr }}\left(\cos \theta^{*}\right)+A_{\text {raw }}^{\text {corr }}\left(-\cos \theta^{*}\right)\right] / 2$. $A_{\text {raw }}^{\text {corr }}$ is the raw asymmetry after being corrected for $A_{\varepsilon}$. The latter is determined with enough precision by using Cabibbo-favored decays, assuming no $\mathrm{CP}$ violation therein.

In this section and the next, we show the prospects in $A_{\mathrm{CP}}$ measurements of $D$ mesons with the target $50 \mathrm{ab}^{-1}$ of Belle II. The existing Belle results will be extrapolated for reducible systematic uncertainties $\left(\sigma_{\text {red }}\right)$ as well as the statistical error $\left(\sigma_{\text {stat }}\right)$. The irreducible systematic errors, such as those due to asymmetric $K^{0}$ vs. $\bar{K}^{0}$ interactions in detector materials and intrinsic CP violations in the $K^{0}$ mesons, etc., are combined separately.

$$
\sigma_{\text {Belle II }}=\sqrt{\left(\sigma_{\text {stat }}^{2}+\sigma_{\text {red }}^{2}\right)^{2} \frac{\mathscr{L}_{\text {Belle }}^{\text {int }}}{50 \mathrm{ab}^{-1}}+\sigma_{\text {irred }}^{2}},
$$

where $\mathscr{L}_{\text {Belle }}^{\text {int }}$ is the integrated luminosity recorded by Belle. In this extrapolation, in the remaining part of this talk, we do not include the effects of detector improvement or ROE tagging.

\section{1 $A_{\mathrm{CP}}\left(D^{0} \rightarrow V \gamma\right)$}

In the $\mathrm{SM}, D^{0} \rightarrow V \gamma$ decays are dominated by long-distance effects, hence providing an excellent test for strong interaction theory [5]. The SM expectations for $A_{\mathrm{CP}}\left(D^{0} \rightarrow V \gamma\right)$ are of the order of $10^{-3}$, but new physics beyond the SM may enhance it to $\mathscr{O}\left(10^{-2}\right)$ [6]. Moreover, there is a recent suggestion that measuring the photon polarization in the time-dependent analysis of radiative charm meson decays can test the SM [8]. Recently Belle has measured the $D^{0} \rightarrow V \gamma$ decays [7], making the first observation of $D^{0} \rightarrow \rho^{0} \gamma$ with $\mathscr{B}\left(D^{0} \rightarrow \rho^{0} \gamma\right)=(1.77 \pm 0.30 \pm 0.77) \times 10^{-5}$, and confirming $D^{0} \rightarrow \phi \gamma$ and $\bar{K}^{* 0} \gamma$. The measured $A_{\mathrm{CP}}$ values are consistent with zero, with statistical errors dominating the uncertainties, for example, $A_{\mathrm{CP}}\left(D^{0} \rightarrow \bar{K}^{* 0} \gamma\right)=-0.003 \pm 0.020 \pm 0.000$.

The dominant background for $D^{0} \rightarrow V \gamma$ is $D \rightarrow V \pi^{0}$ where a photon from $\pi^{0}$ decay is missed. This causes a slight downward shift of $D$ mass peak (see Fig. 4, left), thereby making $M(D)$ resolution a crucial element of analysis. In the study based on Belle II MC, the $\pi^{0}$ veto algorithm performs better than that of Belle (see Fig. 4, middle). Also from Belle II MC studies, the resolutions for both $M(D)$ and $\cos \theta_{\mathrm{H}}$, the helicity angle of $V$ decay particle, are similar to those of Belle (see Fig. 4, right). So we can extrapolate the existing Belle results to the target luminosity of Belle II.

Table 1 shows the expected uncertainty of $A_{\mathrm{CP}}$ in $D^{0} \rightarrow V \gamma$ decays, comparing the recent Belle results [7] with the Belle II expectations, at 5, 15, and $50 \mathrm{ab}^{-1}$ [3], respectively. The Belle II uncertainties are estimated by scaling the Belle results by Eq. 3.1. Roughly $\mathscr{O}(1 \%)$ precision is expected for $A_{\mathrm{CP}}\left(D^{0} \rightarrow V \gamma\right)$ at Belle II, with the statistical error still dominating the total uncertainty.

\section{2 $A_{\mathrm{CP}}\left(D \rightarrow P P^{\prime}\right)$}

The CP asymmetries predicted in the SM for $D \rightarrow P P^{\prime}(P$ is a pseudoscalar meson such as $\pi$, $\eta$, $K$, etc.) modes are also in the $\mathscr{O}\left(10^{-3}\right)$ range. Unlike $D^{0} \rightarrow V \gamma$, however, these decays are not an automatic probe for NP due to uncertainties in hadronic matrix elements. If several different modes are considered together, one can use symmetries, such as flavor $\mathrm{SU}(3)$, to predict and test 

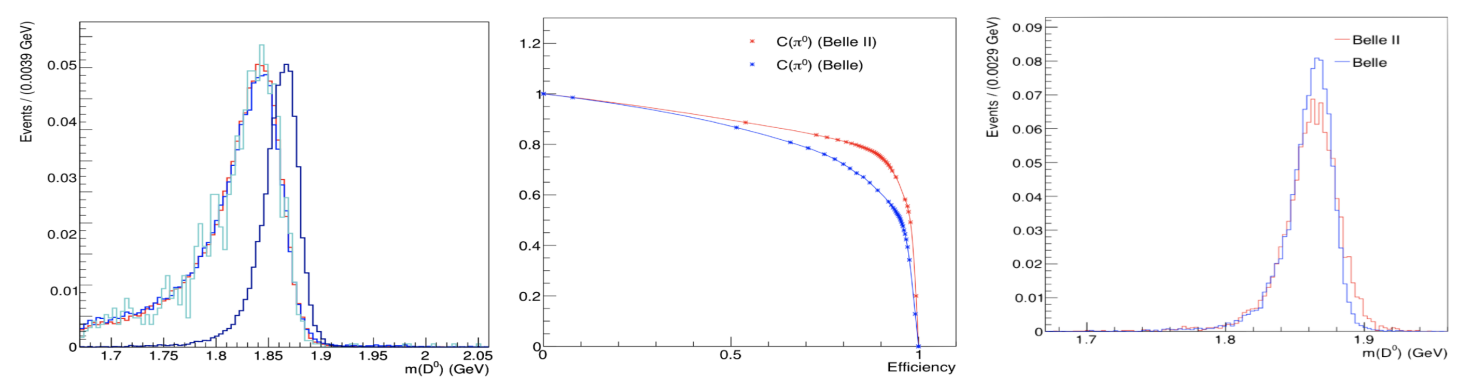

Figure 4: [Left] Comparison of $M\left(D^{0}\right)$ distribution of signal (right-most distribution) and of $\pi^{0}$-type backgrounds, for $D^{0} \rightarrow \bar{K}^{* 0} \gamma$; [Middle] Efficiency vs. background rejection of $\pi^{0}$ veto for Belle II (red) and Belle (blue); [Right] Comparison of $M\left(D^{0}\right)$ of Belle II (red) and Belle (blue) MC samples, for $D^{0} \rightarrow \phi \gamma$.

\begin{tabular}{|c|c|c|c|c|}
\hline \multirow{2}{*}{$\delta A_{\mathrm{CP}}$ on } & Belle & \multicolumn{3}{|c|}{ Belle II (stat. err.) } \\
\cline { 2 - 5 } & $1 \mathrm{ab}^{-1}$ & $5 \mathrm{ab}^{-1}$ & $15 \mathrm{ab}^{-1}$ & $50 \mathrm{ab}^{-1}$ \\
\hline$D^{0} \rightarrow \rho^{0} \gamma$ & $\pm 0.152 \pm 0.006$ & \pm 0.07 & \pm 0.04 & \pm 0.02 \\
$D^{0} \rightarrow \phi \gamma$ & $\pm 0.006 \pm 0.001$ & \pm 0.03 & \pm 0.02 & \pm 0.01 \\
$D^{0} \rightarrow \bar{K}^{* 0} \gamma$ & $\pm 0.020 \pm 0.000$ & \pm 0.01 & \pm 0.005 & \pm 0.003 \\
\hline
\end{tabular}

Table 1: Comparison of $A_{\mathrm{CP}}$ uncertainties of Belle measurements and Belle II expectations for various integrated luminosity milestones, for radiative $D^{0}$ decays.

a pattern among those $A_{\mathrm{CP}}$ measurements. Therefore, it is essential to measure $A_{\mathrm{CP}}$ in as many $D$ decay modes as possible for a comprehensive understanding of $\mathrm{CP}$ asymmetry in the charm system.

Recent measurements of CP asymmetry in $D \rightarrow P P^{\prime}$ includes, for example, $A_{\mathrm{CP}}\left(D^{0} \rightarrow K^{+} K^{-}\right)=$ $(0.04 \pm 0.12 \pm 0.10) \%$ by LHCb [9]. The measurements of the all-charged final-state modes are dominated by $\mathrm{LHCb}$, wherein the statistical and systematic errors are already comparable. For the modes with neutral final-state particles $\left(\pi^{0}, \eta, \eta^{\prime}, K_{S}^{0}\right.$, etc.), which Belle II (and Belle, BaBar, too) can measure well, the statistical error dominates the total uncertainty as shown by the Belle measurements below, and we expect steady improvement with accumulation of the Belle II data.

\subsection{1 $A_{\mathrm{CP}}\left(D^{0} \rightarrow K_{S}^{0} K_{S}^{0}\right)$}

Owing to a large contribution of the exchange diagram process in $D^{0} \rightarrow K_{S}^{0} K_{S}^{0}$, the $A_{\mathrm{CP}}$ in this decay mode can be enhanced to $\mathscr{O}(\%)$. By means of a perturbative calculation estimating penguin annihilation contribution in the SM, one sets an upper limit: $\left|A_{\mathrm{CP}}\left(D^{0} \rightarrow K_{S}^{0} K_{S}^{0}\right)\right|<1.1 \%$ at $95 \%$ confidence level [10]. Therefore, this measurement has a good potential for an early discovery of non-zero $A_{\mathrm{CP}}$ at Belle II. With a data sample of $\mathscr{L}_{\text {int }}=921 \mathrm{fb}^{-1}$, Belle has measured $A_{\mathrm{CP}}\left(D^{0} \rightarrow\right.$ $\left.K_{S}^{0} K_{S}^{0}\right)=(-0.02 \pm 1.53 \pm 0.17) \%$ [11], where the effect of intrinsic CP violation in the neutral kaon system is subtracted. The irreducible systematic uncertainty is of the order of $0.02 \%$. With $50 \mathrm{ab}^{-1}$ at Belle II, we expect $\sigma_{A_{\mathrm{CP}}}=0.23 \%$ [3].

\subsection{2 $A_{\mathrm{CP}}\left(D^{+} \rightarrow \pi^{+} \pi^{0}\right)$}

In the $\mathrm{SM}$ with an isospin symmetry, $A_{\mathrm{CP}}^{\mathrm{SM}}\left(D^{+} \rightarrow \pi^{+} \pi^{0}\right)=0$. Therefore, a non-zero measurement of this can be a smoking gun for NP. With $\mathscr{L}_{\text {int }}=921 \mathrm{fb}^{-1}$ and using $D^{+} \rightarrow K_{S}^{0} \pi^{+}$mode as a reference, Belle has measured $A_{\mathrm{CP}}\left(D^{+} \rightarrow \pi^{+} \pi^{0}\right)=(2.31 \pm 1.24 \pm 0.23) \%$ [12]. Belle II MC 
study using an early version of the reconstruction software shows that the efficiency as well as the background rejection turn out to be similar to those of Belle [3]. We may expect further improvements with an updated software including a better calibration. By scaling the Belle result with the luminosity ratio, the Belle II expectation at $50 \mathrm{ab}^{-1}$ is $\sigma_{A_{\mathrm{CP}}} \approx 0.17 \%$ for this mode.

\section{Time-dependent charm CP violation}

With huge charm production cross-section of high-energy $p p$ collisions, the mixing-induced time-dependent CP violation measurements in the all-charged final-state modes, e.g. $D^{0}\left(\rightarrow \bar{D}^{0}\right) \rightarrow$ $K^{+} \pi^{-}$, will be dominated by LHCb measurements. In this talk, we will focus on a mode where Belle II has an advantage: $D \rightarrow K_{S}^{0} \pi^{+} \pi^{-}$. Being a 3-body decay, $D \rightarrow K_{S}^{0} \pi^{+} \pi^{-}$can be properly studied by analyzing the Dalitz plot distribution. In this case, the most important irreducible systematic error arises from the Dalitz distribution modeling. In the Belle analysis using $921 \mathrm{fb}^{-1}$ [13], the statistical errors dominate the systematic uncertainties by a factor of $(3 \sim 5)$, and the magnitude of reducible and irreducible systematic errors are similar in all the parameters of interest: $x, y,|q / p|$, and $\arg (q / p)$. Extrapolating to $50 \mathrm{ab}^{-1}$, the Belle II expects that the total uncertainties will be highly dominated by the irreducible systematic error (the values within the parenthesis are for irreducible systematic error): $\sigma_{x} \approx 0.11 \%(0.11 \%), \sigma_{y} \approx 0.05 \%(0.04 \%)$, $\sigma_{|q / p|} \approx 7.0-7.4 \%(6.7-7.0 \%), \sigma_{\phi} \approx 4.0-4.2^{\circ}\left(3.7-3.8^{\circ}\right)$ [3]. Consequently, one anticipates to use the model-independent method description, based on the measurements of the strong phase variation across the Dalitz plane. This is currently provided by CLEO-c [14], and improved measurements are expected from BES III [15]. Using the approach based on these measurements will be crucial in order to reduce the systematic uncertainties of the resulting CPV parameters.

\section{Conclusion}

$\mathrm{CP}$ violation in the charm sector is a great place to probe new physics beyond the SM. Belle II is making all-out efforts to fight the systematic uncertainties as well as effectively increasing the sample size, e.g. by improving the tagging algorithm. With the target luminosity of $50 \mathrm{ab}^{-1}$, many interesting results are expected in the charm CP violation. So, please stay tuned!

\section{References}

[1] K. Abe et al. (Belle Collaboration), Phys. Rev. Lett. 87, 091802 (2001).

[2] M. Tanabashi et al. (Particle Data Group), Phys. Rev. D 98, 030001 (2018) and 2019 update.

[3] E. Kou et al., The Belle II Physics Book, Prog. Theo. Exp. Phys. 2019, 123 C01 (2019).

[4] J. P. Lees et al. (BABAR Collaboration), Phys. Rev. D 87, 012004 (2013).

[5] G. Burdman, E. Golowich, J. L. Hewett, and S. Pakvasa, Phys. Rev. D 52, 6383 (1995);

A. Khodjamirian, G. Stoll, and D. Wyler, Phys. Lett. B 358, 129 (1995); S. Fajfer, S. Prelovšek, and P. Singer, Eur. Phys. J. C 6, 471 (1999).

[6] G. Isidori and J. F. Kamenik, Phys. Rev. Lett. 109, 171801 (2012); S. de Boer and G. Hiller, J. High Energy Phys. 1708, 091 (2017). 
[7] T. Nanut et al. (Belle Collaboration), Phys. Rev. Lett. 118, 051801 (2017).

[8] S. de Boer and G. Hiller, Eur. Phys. J. C 78, 188 (2018).

[9] R. Aaij et al. (LHCb Collaboration), Phys. Lett. B 767, 177 (2017).

[10] U. Nierste and S. Schacht, Phys. Rev. D 92, 054036 (2015).

[11] N. Dash et al. (Belle Collaboration), Phys. Rev. Lett. 119, 171801 (2017).

[12] V. Babu et al. (Belle Collaboration), Phys. Rev. D 97, 011101(R) (2018).

[13] T. Peng et al. (Belle Collaboration), Phys. Rev. D 89, 091103(R) (2014).

[14] J. Libby et al. (CLEO Collaboration), Phys. Rev. D 82, 112006 (2010).

[15] Lei Li, Quantum-correlated DD inputs to CKM angle $\gamma$ from BES III, in these proceedings. 